\title{
New Reynolds equation for line contact based on the Carreau model modification by Bair
}

\author{
E. de la Guerra Ochoa , J. Echávarri Otero, E. Chacón Tanarro, P. Lafont Morgado, A. Díaz Lantada, \\ J.M. Munoz-Guijosa, J.L. Muñoz Sanz
}

\begin{abstract}
A B S T R A C T
This paper presents a new form of the one-dimensional Reynolds equation for lubricants whose rheological behaviour follows a modified Carreau rheological model proposed by Bair. The results of the shear stress and flow rate obtained through a new Reynolds-Carreau equation are shown and compared with the results obtained by other researchers.
\end{abstract}

\section{Introduction}

The classic Reynolds formula, Eq. (1), is obtained under the Newtonian fluid hypothesis. It therefore does not include the variation in viscosity $(\eta)$ through the film thickness $(h)$.

$\frac{\partial}{\partial x}\left(\frac{\rho h^{3}}{\eta} \frac{\partial p}{\partial x}\right)=12 u_{m} \frac{\partial(\rho h)}{\partial x}$

For contacts that are highly loaded with small film thickness, which is typical of elastohydrodynamic lubrication (EHL), the fluid ceases to be Newtonian. The use of Reynolds equations that include non-Newtonian effects has led to improvements in the calculation of film thickness $[1,2]$ and in the prediction of the friction coefficient.

The development of generalised Reynolds equations has been frequently based on two-dimensional models [3], such as the one presented in this article. This type of models is generally used in some applications of gears, cams and bearings [4,5].

However, the development of generalised exact Reynolds equations for non-Newtonian fluids has mainly focussed on fluids that fulfil the Eyring rheological model [6-11] or Ellis model [1,6], both of which have their limitations for predicting the variation in viscosity over a wide range of shear rate [12]. For other non-Newtonian liquid models, like the Ostwald-de Waele, Spriggs or Rabinowitsch models, exact Reynolds equations can be found in Ref. [13].

\section{Rheological Carreau model}

The Carreau model [14] is a non-Newtonian model where viscosity depends on shear rate, as follows:

$\eta=\mu\left(1+\left(\frac{\mu \dot{\gamma}}{G}\right)^{2}\right)^{(n-1) / 2}$

When trying to find the generalised Reynolds equation for a non-Newtonian fluid, it is simpler to use a modified Carreau Eq. (3), proposed by Bair $[1,15]$, with $\tau$ as the independent variable. This expression is valid for $0.2<n<1$, which means it can be applied to most non-Newtonian fluids that fit the Carreau model. Otherwise, a numerical calculation would be required to find the velocities field.

$\eta=\mu\left(1+\left(\frac{\tau}{G}\right)^{2}\right)^{(1-1 / n) / 2}$

\section{Reynolds-Carreau equation}

Bair and Khonsari $[13,16]$ have put forward an approximate Reynolds equation adapted to the Carreau model. It is based on a numerical adjustment of the equations for flow rate $(Q)$ and mid-plane shear stress $\left(\tau_{m}\right)$ taken from 103 numerical simulations for different operating conditions. The dimensionless expressions they obtained are as follows for the flow rate and mid-plane shear stress, and will serve for making a comparison 


\section{Nomenclature}

$\alpha \quad$ variable of integration (m)

$\beta \quad$ variable of integration (m)

$\dot{\gamma} \quad$ shear rate $\left(\mathrm{s}^{-1}\right)$

$\eta \quad$ viscosity (Pa s)

$\bar{\eta} \quad$ dimensionless viscosity

$\mu \quad$ low shear viscosity (Pa s)

$\rho$ density $\left(\mathrm{kg} / \mathrm{m}^{3}\right)$

$\Sigma \quad\left(u_{2}-u_{1}\right) / u_{\mathrm{m}}$, slide-to-roll ratio (SRR)

$\tau \quad$ shear stress (Pa)

$\tau_{m} \quad$ mid-plane shear stress (Pa)

$\tau_{m} \quad$ mid-plane dimensionless shear stress

$\bar{\tau}_{\text {m;Bair }}$ mid-plane dimensionless shear stress calculated using analytical formula by Bair

$\bar{\tau}_{\text {m:new }}$ mid-plane dimensionless shear stress calculated using new analytical formula proposed in this article mid-plane dimensionless shear stress calculated using numerical simulation by Bair

G

\begin{tabular}{|c|c|}
\hline$h$ & film thickness (m) \\
\hline$n$ & carreau exponent \\
\hline $\boldsymbol{p}$ & pressure $(\mathrm{Pa})$ \\
\hline $\bar{p}^{\prime}$ & $(h / G)(\partial p / \partial x)$, dimensionless pressure gradient \\
\hline$Q$ & flow rate per unit length $\left(\mathrm{m}^{2} / \mathrm{s}\right)$ \\
\hline $\bar{Q}$ & dimensionless flow rate \\
\hline $\bar{Q}_{\text {Bair }}$ & $\begin{array}{l}\text { dimensionless flow rate calculated using the analyti- } \\
\text { cal formula by Bair }\end{array}$ \\
\hline $\bar{Q}_{\text {new }}$ & $\begin{array}{l}\text { dimensionless flow rate calculated using the new } \\
\text { analytical formula proposed in this article }\end{array}$ \\
\hline $\bar{Q}_{\text {sim }}$ & $\begin{array}{l}\text { dimensionless flow rate calculated using numerical } \\
\text { simulation by Bair }\end{array}$ \\
\hline$u$ & velocity $(\mathrm{m} / \mathrm{s})$ \\
\hline$u_{m}$ & average velocity $(\mathrm{m} / \mathrm{s})$ \\
\hline$u_{i}$ & velocity of solid $i(i=1,2)(\mathrm{m} / \mathrm{s})$ \\
\hline $\bar{u}$ & dimensionless velocity \\
\hline $\bar{u}_{m}$ & dimensionless mean velocity \\
\hline $\bar{u}_{i}$ & dimensionless velocity of solid $i$ \\
\hline$x$ & coordinate in flow direction $(\mathrm{m})$ \\
\hline$z$ & coordinate across flow direction (m) \\
\hline
\end{tabular}

with the new equations obtained in this article:

$$
\begin{aligned}
\bar{\tau}_{m} & =\sum \bar{u}_{m}\left(1+0.53 \bar{p}^{\prime 4}\right)^{[1-(1 / n)] / 4}\left[1+\left(\sum \bar{u}_{m}\right)^{2}\right]^{(n-1) / 2} \\
\bar{Q} & =\left\{\begin{array}{c}
\bar{u}_{m}-\bar{p}_{12}^{\prime}+0.57 \frac{(n-1)}{(1+(1 / n)) \bar{p}}\left|\bar{p}_{2}^{\prime(1 / n)+(4 / 3)}\right|^{\text {or }} \\
\bar{u}_{m}\left[(1+0.0047)\left(\left|\bar{p}^{\prime}\right|^{2.72}\left|\bar{\tau}_{m}\right|\right)\right]^{1-(1 / n)}-\frac{\bar{p}^{\prime}}{12}+0.57 \frac{(n-1)}{(1+(1 / n)) \bar{p}}\left|\frac{\bar{p}^{\prime}}{2}\right|^{(1 / n)+(4 / 3)}
\end{array}\right.
\end{aligned}
$$

These equations are fitted for the following dimensionless operating ranges:

$n=0.3,0.5$ and 0.75

$0.01 \leq \bar{u}_{m} \leq 1$ and $\bar{u}_{m}=-1$

$-27 \leq \bar{p}^{\prime} \leq 27$

$-0.5 \leq \Sigma \leq 1$

\section{New Reynolds-Carreau equation}

The procedure followed is identical to that for finding the Newtonian Reynolds equation. The first step is to balance the forces of an element of the contacting fluid, as Fig. 1 shows.

This gives

$\frac{\partial p}{\partial x}=\frac{\partial \tau}{\partial z}$

To obtain the Reynolds equation the velocities field needs to be calculated. The field can be defined by taking the integral expression as follows, where the origin of $z$ is the mean point of the film thickness $(-h / 2<z<h / 2)$ :

$u(z)=u_{1}+\int_{-h / 2}^{z} \frac{\partial u}{\partial z} d z$

Inserting the relationship between viscosity $(\eta)$ and shear stress $(\tau)$ into the velocity gradient gives Eq. (8)

$u=u_{1}+\int_{-h / 2}^{z} \frac{\tau}{\eta} d z=u_{1}+\frac{1}{\mu} \int_{-h / 2}^{z} \tau\left(1+\left(\frac{\tau}{G}\right)^{2}\right)^{[(1 / n)-1] / 2} d z$
By taking the balance of forces obtained in Eq. (6) for $\frac{\partial p}{\partial x} \neq 0$ and making a substitution in the integration variable

$\frac{\partial p}{\partial x}=\frac{\partial \tau}{\partial z} \Rightarrow \tau=\tau_{m}+z \frac{\partial p}{\partial x} \Rightarrow d \tau=d z \frac{\partial p}{\partial x}$

where $\tau_{m}$ is defined as the value of the shear stress at point $z=0$. By making the corresponding change in the integration limits, expression (8) is transformed to

$u=u_{1}+\frac{1}{\mu(\partial p / \partial x)} \int_{\alpha}^{\tau} \tau\left(1+\left(\frac{\tau}{G}\right)^{2}\right)^{[(1 / n)-1] / 2} d \tau$

where $\alpha=\tau_{m}-\frac{\partial p h}{\partial \times \frac{2}{2}}$

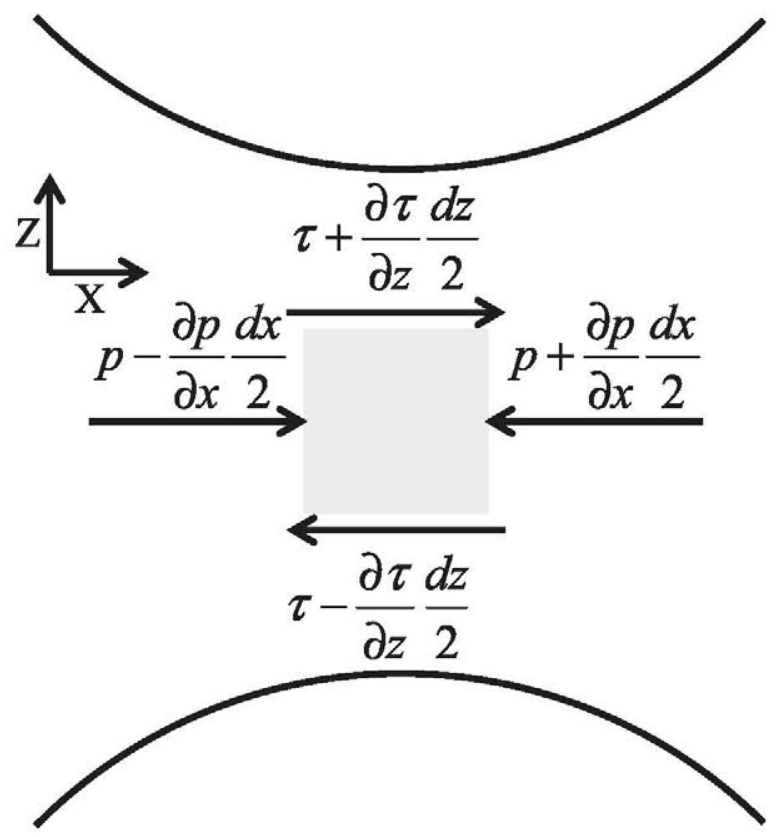

Fig. 1. Line contact diagram. 
Integrating Eq. (10) with $\tau>0$ gives the fluid velocity field across the film thickness:

$u=u_{1}+\frac{1}{\mu(\partial p / \partial x)}\left[\frac{G^{1-(1 / n)} n\left(\left(G^{2}+\tau^{2}\right)^{(1+n) / 2 n}-\left(G^{2}+\alpha^{2}\right)^{(1+n) / 2 n)}\right.}{1+n}\right]$

Transforming back to terms of $z$ and developing leads to:

$$
\begin{aligned}
u= & u_{1}+\frac{1}{\mu(\partial p / \partial x)} \frac{G^{1-(1 / n)} n}{1+n}\left[\left(G^{2}+\tau_{m}^{2}+2 \tau_{m} z \frac{\partial p}{\partial x}+\left(z \frac{\partial p}{\partial x}\right)^{2}\right)^{(1+n) / 2 n}\right. \\
& \left.-\left(G^{2}+\tau_{m}^{2}-\tau_{m} h \frac{\partial p}{\partial x}+\frac{1}{4}\left(h \frac{\partial p}{\partial x}\right)^{2}\right)^{(1+n) / 2 n}\right]
\end{aligned}
$$

By particularising $u$ for $z=h / 2$, Eq. (14) is obtained. This expression provides the value of $\tau_{m}$, which a priori was unknown.

$$
\begin{gathered}
\frac{\left(u_{2}-u_{1}\right) \mu(\partial p / \partial x)(1+n)}{G^{1-(1 / n)} n}=\left(G^{2}+\tau_{m}^{2}+\tau_{m} h \frac{\partial p}{\partial x}+\frac{1}{4}\left(h \frac{\partial p}{\partial x}\right)^{2}\right)^{(1+n) / 2 n} \\
-\left(G^{2}+\tau_{m}^{2}-\tau_{m} h \frac{\partial p}{\partial x}+\frac{1}{4}\left(h \frac{\partial p}{\partial x}\right)^{2}\right)^{(1+n) / 2 n}
\end{gathered}
$$

This expression (14) has no analytical solution in general. Therefore, in order to find the value of $\tau_{m}$ numerical calculation is required. In this case, the Newton-Raphson method is used.

The next step is to calculate the flow rate per unit length by integrating the velocities field:

$$
\begin{aligned}
Q= & \int_{-h / 2}^{h / 2}\left(u_{1}+\frac{1}{\mu(\partial p / \partial x)} \frac{G^{1-(1 / n)} n}{1+n}\left[\left(G^{2}+\tau_{m}^{2}+2 \tau_{m} z \frac{\partial p}{\partial x}+\left(z \frac{\partial p}{\partial x}\right)^{2}\right)^{(1+\pi) / 2 n}\right.\right. \\
& \left.\left.-\left(G^{2}+\tau_{m}^{2}-\tau_{m} h \frac{\partial p}{\partial x}+\frac{1}{4}\left(h \frac{\partial p}{\partial x}\right)^{2}\right)^{(1+n) / 2 n}\right]\right) d z
\end{aligned}
$$

By again making the same substitution in the integration variable, Eq. (9), the following equation is obtained:

$$
\begin{aligned}
Q= & \int_{\alpha}^{\beta} \frac{1}{(\partial p / \partial x)} \\
& \times\left(u_{1}+\frac{1}{\mu(\partial p / \partial x)}\left[\frac{G^{1-1 / n} n\left(\left(G^{2}+\tau^{2}\right)^{(1+n) / 2 n}-\left(G^{2}+\alpha^{2}\right)^{(1+n) / 2 n}\right)}{1+n}\right]\right) d \tau
\end{aligned}
$$

where $\beta$ is given by the expression:

$\beta=\tau_{m}+\frac{\partial p}{\partial x} \frac{h}{2}$

Under the following integration rule, obtained through Maple's symbolic integration modulus (where the integrand is positive) the solution can be calculated:

$$
\begin{gathered}
\int\left(G^{1-(1 / n)} n\left(G^{2}+\tau^{2}\right)^{(1+n) / 2 n}\right) d \tau=n G^{2} \tau \cdot{ }_{2} F_{1}\left(\left[\frac{1}{2},-\frac{1+n}{2 n}\right],\left[\frac{3}{2}\right],-\frac{\tau^{2}}{G^{2}}\right) \\
=n G^{2} \tau \text { hypergeom }\left(\left[\frac{1}{2},-\frac{1+n}{2 n}\right],\left[\frac{3}{2}\right],-\frac{\tau^{2}}{G^{2}}\right)
\end{gathered}
$$

where ${ }_{2} F_{1}$ is the Gauss hypergeometric function [14], that corresponds to a particular case of the generalised form of the hypergeometric function or Barnes extended hypergeometric function. It is denoted by ${ }_{j} F_{k}(a, b, z)$, where $j$ is the length of vector $a$ and $k$ is the length of vector $b$. For further clarity the following notation is used in this paper: hypergeom $\left(\left[a_{1}, a_{2}, \ldots\right]\right.$, $\left.\left[b_{1}, b_{2}, \ldots\right], z\right)[17,18]$.
By developing expression (16) and simplifying, we obtain the expression for the flow rate:

$$
\begin{aligned}
Q= & u_{1} h-\frac{G^{(n-1) / n} n\left(G^{2}+\left(\tau_{m}-(\partial p / \partial x)(h / 2)\right)^{2}\right)^{(1+n) / 2 n} h}{(1+n) \mu(\partial p / \partial x)} \\
& +\frac{n G^{2}\left(\tau_{m}+(\partial p / \partial x)(h / 2)\right)}{(1+n) \mu(\partial p / \partial x)^{2}} \times \text { hypergeom } \\
& \left(\left[\frac{1}{2},-\frac{1+n}{2 n}\right],\left[\frac{3}{2}\right],-\frac{\left(\tau_{m}+(\partial p / \partial x)(h / 2)\right)^{2}}{G^{2}}\right) \\
& -\frac{n G^{2}\left(\tau_{m}-(\partial p / \partial x)(h / 2)\right)}{(1+n) \mu(\partial p / \partial x)^{2}} \times \text { hypergeom } \\
& \left(\left[\frac{1}{2},-\frac{1+n}{2 n}\right],\left[\frac{3}{2}\right],-\frac{\left(\tau_{m}-(\partial p / \partial x)(h / 2)\right)^{2}}{G^{2}}\right)
\end{aligned}
$$

Applying the law of conservation of mass will give the ReynoldsCarreau analytical equation:

$$
\begin{aligned}
& \frac{d(\rho Q)}{d x}=0
\end{aligned}
$$

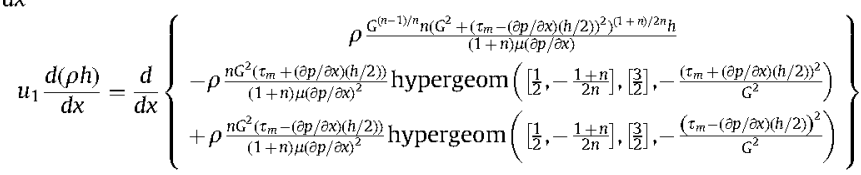

The resulting expression (20) is a function of $\tau_{m}$, which means that to find the flow rate, all that is required is to insert the values of $\tau_{m}$ calculated from Eq. (14).

So that the results from the equations obtained can be compared with those found by Bair-Khonsari, Eq. (20) is made dimensionless with the same dimensionless parameters used by Bair-Khonsari. These parameters are

$$
\begin{gathered}
\bar{\tau}=\frac{\tau}{G}, \quad \bar{u}_{i}=\frac{u_{i} \mu}{h G}, \quad \bar{z}=\frac{z}{h}, \quad \bar{\eta}=\frac{\eta}{\mu}, \quad \bar{p}^{\prime}=p^{\prime} \frac{h}{G}, \\
\bar{Q}=\frac{Q \mu}{h^{2} G}, \quad \Sigma=\frac{\left(u_{2}-u_{1}\right)}{u_{m}}
\end{gathered}
$$

By inserting these parameters in Eqs. (3), (13), (14) and (19), dimensionless equation is obtained for the Carreau model, the mid-plane shear stress, the flow rate and the Reynolds-Carreau equation:

$$
\begin{aligned}
& \bar{\eta}=\left(1+\bar{\tau}^{2}\right)^{[1-(1 / n)] / 2} \\
& \bar{u}=\bar{u}_{1}+\frac{n}{(1+n)} \frac{1}{\bar{p}^{\prime}}\left[\left(1+\bar{\tau}_{m}^{2}+2 \bar{\tau}_{m} \overline{z p^{\prime}}+\left(\overline{z p^{\prime}}\right)^{2}\right)^{(1+n) / 2 n}\right. \\
& \left.-\left(1+\bar{\tau}_{m}^{2}-\bar{\tau}_{m} \bar{p}^{\prime}+\frac{1}{4} \bar{p}^{2}\right)^{(1+n) / 2 n}\right] \\
& \left(\bar{u}_{2}-\bar{u}_{1}\right) \frac{(1+n)}{n} \bar{p}^{\prime}=\left[\left(1+\bar{\tau}_{m}^{2}+\bar{\tau}_{m} \bar{p}^{\prime}+\frac{1}{4} \bar{p}^{2}\right)^{(1+n) / 2 n}\right. \\
& \left.-\left(1+\bar{\tau}_{m}^{2}-\bar{\tau}_{m} \bar{p}^{\prime}+\frac{1}{4} \bar{p}^{2}\right)^{(1+n) / 2 n}\right] \\
& \overline{\mathrm{Q}}=\left\{\begin{array}{c}
\bar{u}_{1}-\frac{n}{\bar{p}^{\prime}(1+n)}\left(1+\left(\bar{\tau}_{m}-\frac{\bar{p}^{\prime}}{2}\right)^{2}\right)^{(1+n) / 2 n} \\
+\frac{n}{\bar{p}^{\prime 2}(\mathbf{1}+n)}\left(\bar{\tau}_{m}+\frac{\bar{p}^{\prime}}{2}\right) \text { hypergeom }\left(\left[\frac{1}{2},-\frac{1+n}{2 n}\right],\left[\frac{3}{2}\right],-\left(\bar{\tau}_{m}+\frac{\bar{p}^{\prime}}{2}\right)^{2}\right) \\
-\frac{n}{\bar{p}^{2}(1+n)}\left(\bar{\tau}_{m}-\frac{\bar{p}^{\prime}}{2}\right) \text { hypergeom }\left(\left[\frac{1}{2},-\frac{1+n}{2 n}\right],\left[\frac{3}{2}\right],-\left(\bar{\tau}_{m}-\frac{\bar{p}^{\prime}}{2}\right)^{2}\right)
\end{array}\right.
\end{aligned}
$$



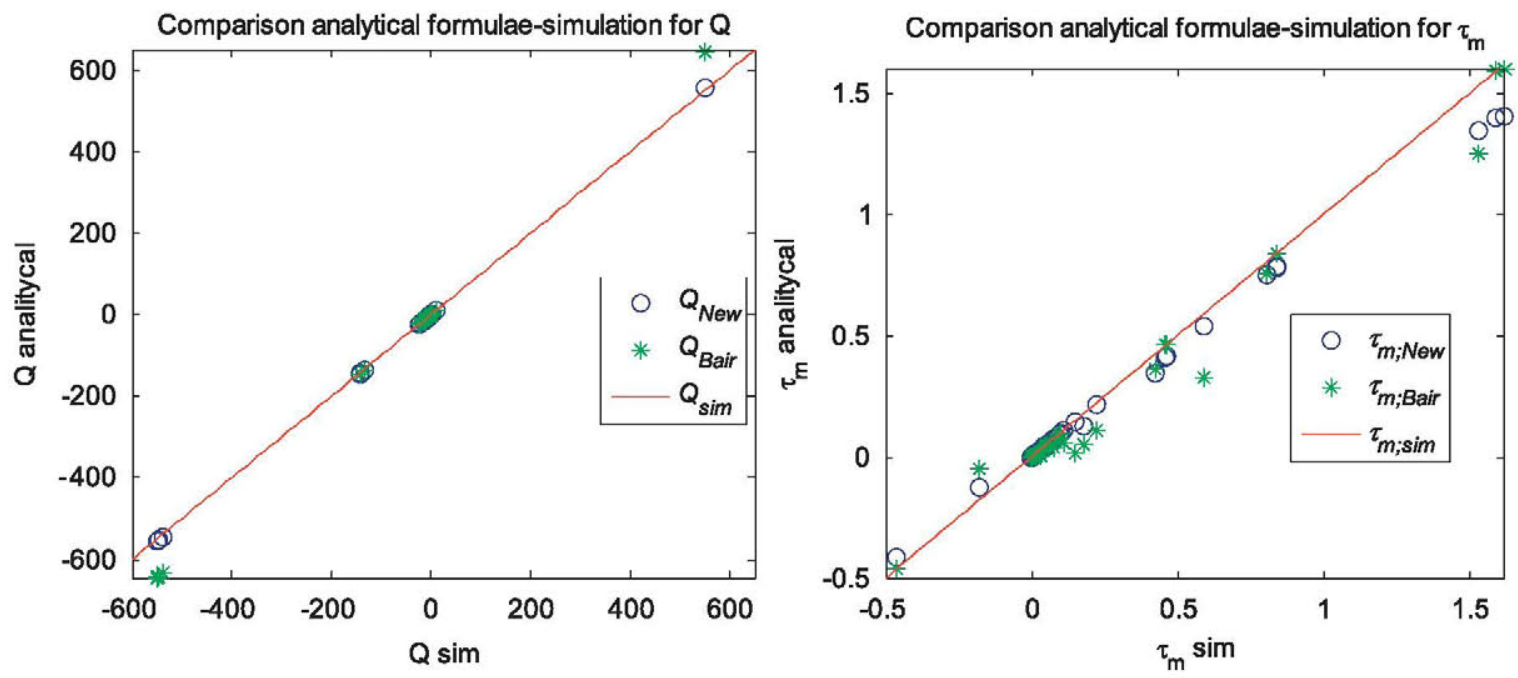

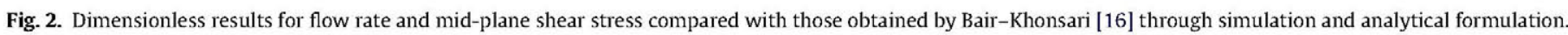

\section{Results obtained with the Carreau model modification by Bair}

Having presented the new equations, we will now compare the results for the flow rate and mid-plane shear stress obtained for different operating conditions with those obtained by BairKhonsari through their equations and simulations.

The appendix shows different operating conditions $\left(\bar{u}_{m}, \Sigma, \bar{p}^{\prime}\right.$ and $n$ ) together with the results obtained by Bair-Khonsari [16], $\left(\bar{Q}_{\text {sim }}\right.$ and $\bar{Q}_{\text {Bair }}, \bar{\tau}_{m ; \text { sim }}$ and $\left.\bar{\tau}_{m ; B a i r}\right)$, and the new results $\left(\bar{Q}_{\text {new }}\right.$ and $\bar{\tau}_{\text {m;new }}$ ) through Eq. (22).

Fig. 2 shows the results obtained in graphic form. The values calculated through analytical expressions (5) and (22) are shown compared with the values obtained through simulations, which are represented by the line $(y=x)$.

The errors obtained are less than $6 \%$ for the values of $\bar{\tau}_{m}$ and less than $8 \%$ for the values of $\bar{Q}_{n e w}$ in all conditions. If a comparison is made with the data obtained by Bair-Khonsari [16], the errors for flow rate are of the same order, but the results for mid-plane shear stress over the whole simulated range are generally better.

\section{Particularisation for the Ellis and Rabinowitsch models}

After verifying the new equations, they are particularised for $n=1 / 3$ where the Carreau model is equivalent to Rabinowitsch and Ellis equations [1]. For these two models analytical results of the Reynolds equation are published in Refs. [1,13,19],and therefore the results derived from the new equations can be compared with the existing equations for this particular case.

By substituting $n=1 / 3$ in (3) and (14) the particular rheological model (23) is found, whereas Eq. (24) gives the shear stress in the mid-plane, $\tau_{m}$.

$\eta=\mu\left(1+\left(\frac{\tau}{G}\right)^{2}\right)^{-1}$

$4\left(u_{2}-u_{1}\right) \mu \frac{\partial p}{\partial x} G^{2}=4 \tau_{m} G^{2} h \frac{\partial p}{\partial x}+\left(\left(\tau_{m}+\frac{h}{2} \frac{\partial p}{\partial x}\right)^{4}-\left(\tau_{m}-\frac{h}{2} \frac{\partial p}{\partial x}\right)^{4}\right)$

In order to verify the flow rate equation, expression (18) particularised to $n=1 / 3$ leads to the polynomial

$$
\begin{gathered}
n G^{2} \tau \text { hypergeom }\left.\left(\left[\frac{1}{2},-\frac{1+n}{2 n}\right],\left[\frac{3}{2}\right],-\frac{\tau^{2}}{G^{2}}\right)\right|_{n=1 / 3} \\
=\frac{1}{3} G^{2} \tau\left(1-\frac{2}{3}\left(-\frac{\tau^{2}}{G^{2}}\right)+\frac{1}{5}\left(-\frac{\tau^{2}}{G^{2}}\right)^{2}\right)
\end{gathered}
$$

By inserting Eq. (25) into (19) and developing, flow rate expression (26) is obtained

$$
\begin{aligned}
Q= & u_{1} h+\frac{\tau_{m} h^{2}}{2 \mu}-\frac{\partial p}{\partial x} \frac{h^{3}}{12 \mu}+\frac{1}{4 G^{2} \mu(\partial p / \partial x)^{2}} \\
& \times\left(\frac{\left(\tau_{m}+(h / 2)(\partial p / \partial x)\right)^{5}}{5}-\frac{\left(\tau_{m}-(h / 2)(\partial p / \partial x)\right)^{5}}{5}-\frac{h}{2} \frac{\partial p}{\partial x}\left(\tau_{m}-\frac{h}{2} \frac{\partial p}{\partial x}\right)^{4}\right)
\end{aligned}
$$

If Eqs. (24) and (26) are developed to deduce the mid-plane shear stress and flow rate, a complete equivalency is found with the results presented by Bair [1,13] when regarding the Rabinowitsch model and the particular case of the Ellis model when $n=1 / 3$.

\section{Conclusions}

This article proposes a new Reynolds-Carreau equation for line contact and expressions for the mid-plane shear stress and flow rate. The equations have been obtained in a general way, unlike the approximate expressions published previously for the Carreau model, which are obtained by fitting the simulations to specific conditions. Therefore, the new equations are applicable to a wider range of conditions.

The new formulae are analytical, simple and easy to implement since the hypergeometric function can be found in most programming libraries (Matlab, Mathematica, Maple among others) and moreover the results obtained with new equations show a good correlation with simulations presented by BairKhonsari [16].

In addition, the presented equations have been particularised for $n=1 / 3$ in order to obtain comparable expressions to the Ellis and Rabinowitsch models. As analytical equations are available for the Ellis and Rabinowitsch models, it has been verified that the particular solution attained by using the new equations is identical to that presented previously.

The Carreau model describes the behaviour of a large number of lubricants and we hope that the equations deduced will be of help in calculating friction and film thickness in line contacts under elastohydrodynamic lubrication. Moreover, the use of analytical equations facilitates the solving process because it avoids numerical integration of the velocity field of the fluid, unlike numerous generalised Reynolds equations where flow factors are used $[6,20,21]$. 


\section{Appendix}

See Table A.1

Table A.1

Summary of results.

\begin{tabular}{|c|c|c|c|c|c|c|c|c|c|}
\hline $\bar{u}_{m}$ & $\Sigma$ & $\bar{p}^{\prime}$ & $n$ & $\bar{Q}_{\text {sim }}$ & $\bar{Q}_{\text {Bair }}$ & $\bar{Q}_{n e w}$ & $\bar{\tau}_{m ; s i m}$ & $\bar{\tau}_{m ; \text { Bair }}$ & $\bar{\tau}_{\text {m:new }}$ \\
\hline 0.1 & 0 & 0.111 & 0.3 & $9.07 \mathrm{E}-02$ & $9.075 \mathrm{E}-02$ & $9.073 \mathrm{E}-02$ & 0 & 0 & 0 \\
\hline 0.1 & 0 & 0.333 & 0.3 & $7.20 \mathrm{E}-02$ & $7.219 \mathrm{E}-02$ & $7.171 \mathrm{E}-02$ & 0 & 0 & 0 \\
\hline 0.1 & 0 & 1 & 0.3 & $1.19 \mathrm{E}-02$ & $1.304 \mathrm{E}-02$ & $1.877 \mathrm{E}-03$ & 0 & 0 & 0 \\
\hline 0.1 & 0 & 3 & 0.3 & $-3.69 \mathrm{E}-01$ & $-3.536 \mathrm{E}-01$ & $-5.817 \mathrm{E}-01$ & 0 & 0 & 0 \\
\hline 0.1 & 0 & 9 & 0.3 & $-1.40 \mathrm{E}+01$ & $-1.208 \mathrm{E}+01$ & $-1.532 \mathrm{E}+01$ & 0 & 0 & 0 \\
\hline 0.1 & 0 & 27 & 0.3 & $-5.49 \mathrm{E}+02$ & $-6.444 \mathrm{E}+02$ & $-5.548 \mathrm{E}+02$ & 0 & 0 & 0 \\
\hline 0.1 & 0 & 18 & 0.3 & $-1.42 \mathrm{E}+02$ & $-1.466 \mathrm{E}+02$ & $-1.453 \mathrm{E}+02$ & 0 & 0 & 0 \\
\hline 0.1 & 0.5 & 0.111 & 0.3 & $9.10 \mathrm{E}-02$ & $9.075 \mathrm{E}-02$ & $9.065 \mathrm{E}-02$ & $5.00 \mathrm{E}-02$ & $4.995 \mathrm{E}-02$ & $4.968 \mathrm{E}-02$ \\
\hline 0.1 & 0.5 & 0.333 & 0.3 & $7.23 \mathrm{E}-02$ & $7.219 \mathrm{E}-02$ & $7.148 \mathrm{E}-02$ & $4.96 \mathrm{E}-02$ & $4.977 \mathrm{E}-02$ & $4.830 \mathrm{E}-02$ \\
\hline 0.1 & 0.5 & 1 & 0.3 & $1.20 \mathrm{E}-02$ & $1.304 \mathrm{E}-02$ & $1.429 \mathrm{E}-03$ & $4.55 \mathrm{E}-02$ & $3.898 \mathrm{E}-02$ & $3.849 \mathrm{E}-02$ \\
\hline 0.1 & 0.5 & 3 & 0.3 & $-3.69 \mathrm{E}-01$ & $-3.536 \mathrm{E}-01$ & $-5.819 \mathrm{E}-01$ & $1.82 \mathrm{E}-02$ & $5.499 \mathrm{E}-03$ & $1.264 \mathrm{E}-02$ \\
\hline 0.1 & 0.5 & 9 & 0.3 & $-1.40 \mathrm{E}+01$ & $-1.208 \mathrm{E}+01$ & $-1.532 \mathrm{E}+01$ & $9.90 \mathrm{E}-04$ & $4.293 \mathrm{E}-04$ & $1.414 \mathrm{E}-03$ \\
\hline 0.1 & 0.5 & 27 & 0.3 & $-5.49 \mathrm{E}+02$ & $-6.444 \mathrm{E}+02$ & $-5.548 \mathrm{E}+02$ & $2.90 \mathrm{E}-04$ & $3.308 \mathrm{E}-05$ & $1.145 \mathrm{E}-04$ \\
\hline 0.1 & 0.5 & 18 & 0.3 & $-1.42 \mathrm{E}+02$ & $-1.466 \mathrm{E}+02$ & $-1.453 \mathrm{E}+02$ & $2.60 \mathrm{E}-04$ & $8.520 \mathrm{E}-05$ & $2.925 \mathrm{E}-04$ \\
\hline 1 & 0 & 0.111 & 0.3 & $9.90 \mathrm{E}-01$ & $9.907 \mathrm{E}-01$ & $9.907 \mathrm{E}-01$ & 0 & 0 & 0 \\
\hline 1 & 0 & 0.333 & 0.3 & $9.72 \mathrm{E}-01$ & $9.722 \mathrm{E}-01$ & $9.717 \mathrm{E}-01$ & 0 & 0 & 0 \\
\hline 1 & 0 & 1 & 0.3 & $9.12 \mathrm{E}-01$ & $9.130 \mathrm{E}-01$ & $9.019 \mathrm{E}-01$ & 0 & 0 & 0 \\
\hline 1 & 0 & 3 & 0.3 & $5.31 \mathrm{E}-01$ & $5.464 \mathrm{E}-01$ & $3.183 \mathrm{E}-01$ & 0 & 0 & 0 \\
\hline 1 & 0 & 9 & 0.3 & $-1.31 \mathrm{E}+01$ & $-1.118 \mathrm{E}+01$ & $-1.442 \mathrm{E}+01$ & 0 & 0 & 0 \\
\hline 1 & 0 & 27 & 0.3 & $-5.48 \mathrm{E}+02$ & $-6.435 \mathrm{E}+02$ & $-5.539 \mathrm{E}+02$ & 0 & 0 & 0 \\
\hline 1 & 0 & 18 & 0.3 & $-1.41 \mathrm{E}+02$ & $-1.457 \mathrm{E}+02$ & $-1.444 \mathrm{E}+02$ & 0 & 0 & 0 \\
\hline 1 & 0.5 & 0.111 & 0.3 & $9.91 \mathrm{E}-01$ & $9.907 \mathrm{E}-01$ & $9.850 \mathrm{E}-01$ & $4.62 \mathrm{E}-01$ & $4.624 \mathrm{E}-01$ & $4.143 \mathrm{E}-01$ \\
\hline 1 & 0.5 & 0.333 & 0.3 & $9.67 \mathrm{E}-01$ & $9.722 \mathrm{E}-01$ & $9.552 \mathrm{E}-01$ & $4.58 \mathrm{E}-01$ & $4.607 \mathrm{E}-01$ & $4.067 \mathrm{E}-01$ \\
\hline 1 & 0.5 & 1 & 0.3 & $8.93 \mathrm{E}-01$ & $9.130 \mathrm{E}-01$ & $8.653 \mathrm{E}-01$ & $4.21 \mathrm{E}-01$ & $3.608 \mathrm{E}-01$ & $3.457 \mathrm{E}-01$ \\
\hline 1 & 0.5 & 3 & 0.3 & $5.05 \mathrm{E}-01$ & $5.464 \mathrm{E}-01$ & $3.014 \mathrm{E}-01$ & $1.78 \mathrm{E}-01$ & $5.091 \mathrm{E}-02$ & $1.256 \mathrm{E}-01$ \\
\hline 1 & 0.5 & 9 & 0.3 & $-1.31 \mathrm{E}+01$ & $-1.118 \mathrm{E}+01$ & $-1.442 \mathrm{E}+01$ & $1.50 \mathrm{E}-02$ & $3.974 \mathrm{E}-03$ & $1.414 \mathrm{E}-02$ \\
\hline 1 & 0.5 & 27 & 0.3 & $-5.48 \mathrm{E}+02$ & $-6.435 \mathrm{E}+02$ & $-5.539 \mathrm{E}+02$ & $1.18 \mathrm{E}-03$ & $3.062 \mathrm{E}-04$ & $1.145 \mathrm{E}-03$ \\
\hline 1 & 0.5 & 18 & 0.3 & $-1.41 \mathrm{E}+02$ & $-1.457 \mathrm{E}+02$ & $-1.444 \mathrm{E}+02$ & $2.20 \mathrm{E}-03$ & 7.887E-04 & $2.925 \mathrm{E}-03$ \\
\hline 10 & 0 & 0.111 & 0.3 & $9.99 \mathrm{E}+00$ & $9.991 \mathrm{E}+00$ & $9.991 \mathrm{E}+00$ & 0 & 0 & 0 \\
\hline 10 & 0 & 0.333 & 0.3 & $9.98 \mathrm{E}+00$ & $9.972 \mathrm{E}+00$ & $9.972 \mathrm{E}+00$ & 0 & 0 & 0 \\
\hline 10 & 0 & 1 & 0.3 & $9.92 \mathrm{E}+00$ & $9.913 \mathrm{E}+00$ & $9.902 \mathrm{E}+00$ & 0 & 0 & 0 \\
\hline 10 & 0 & 3 & 0.3 & $9.53 \mathrm{E}+00$ & $9.546 \mathrm{E}+00$ & $9.318 \mathrm{E}+00$ & 0 & 0 & 0 \\
\hline 10 & 0 & 9 & 0.3 & $-4.12 \mathrm{E}+00$ & $-2.185 \mathrm{E}+00$ & $-5.416 \mathrm{E}+00$ & 0 & 0 & 0 \\
\hline 10 & 0 & 27 & 0.3 & $-5.39 \mathrm{E}+02$ & $-6.345 \mathrm{E}+02$ & $-5.449 \mathrm{E}+02$ & 0 & 0 & 0 \\
\hline 10 & 0 & 18 & 0.3 & $-1.32 \mathrm{E}+02$ & $-1.367 \mathrm{E}+02$ & $-1.354 \mathrm{E}+02$ & 0 & 0 & 0 \\
\hline 10 & 0.5 & 0.111 & 0.3 & $9.94 \mathrm{E}+00$ & $9.991 \mathrm{E}+00$ & $9.916 \mathrm{E}+00$ & $1.62 \mathrm{E}+00$ & $1.598 \mathrm{E}+00$ & $1.403 \mathrm{E}+00$ \\
\hline 10 & 0.5 & 0.333 & 0.3 & $9.67 \mathrm{E}+00$ & $9.972 \mathrm{E}+00$ & $9.750 \mathrm{E}+00$ & $1.59 \mathrm{E}+00$ & $1.593 \mathrm{E}+00$ & $1.397 \mathrm{E}+00$ \\
\hline 10 & 0.5 & 1 & 0.3 & $9.29 \mathrm{E}+00$ & $9.913 \mathrm{E}+00$ & $9.279 \mathrm{E}+00$ & $1.53 \mathrm{E}+00$ & $1.247 \mathrm{E}+00$ & $1.346 \mathrm{E}+00$ \\
\hline 10 & 0.5 & 9 & 0.3 & $-4.20 \mathrm{E}+00$ & $-2.185 \mathrm{E}+00$ & $-5.503 \mathrm{E}+00$ & 1.49E-01 & $1.374 \mathrm{E}-02$ & $1.412 \mathrm{E}-01$ \\
\hline 10 & 0.5 & 27 & 0.3 & $-5.39 \mathrm{E}+02$ & $-6.345 \mathrm{E}+02$ & $-5.449 \mathrm{E}+02$ & $9.90 \mathrm{E}-03$ & $1.059 \mathrm{E}-03$ & $1.145 \mathrm{E}-02$ \\
\hline 10 & 0.5 & 18 & 0.3 & $-1.32 \mathrm{E}+02$ & $-1.367 \mathrm{E}+02$ & $-1.355 \mathrm{E}+02$ & $2.90 \mathrm{E}-02$ & $2.726 \mathrm{E}-03$ & $2.925 \mathrm{E}-02$ \\
\hline-1 & 0 & 0.111 & 0.3 & $-1.01 \mathrm{E}+00$ & $-1.009 \mathrm{E}+00$ & $-1.009 \mathrm{E}+00$ & 0 & 0 & 0 \\
\hline-1 & 0 & 3 & 0.3 & $-1.47 \mathrm{E}+00$ & $-1.454 \mathrm{E}+00$ & $-1.682 \mathrm{E}+00$ & 0 & 0 & 0 \\
\hline-1 & 0 & 27 & 0.3 & $-5.50 \mathrm{E}+02$ & $-6.455 \mathrm{E}+02$ & $-5.559 \mathrm{E}+02$ & 0 & 0 & 0 \\
\hline 1 & 0 & -0.111 & 0.3 & $1.009 \mathrm{E}+00$ & $1.009 \mathrm{E}+00$ & $1.009 \mathrm{E}+00$ & 0 & 0 & 0 \\
\hline 1 & 0 & -3 & 0.3 & $1.47 \mathrm{E}+00$ & $1.454 \mathrm{E}+00$ & $1.682 \mathrm{E}+00$ & 0 & 0 & 0 \\
\hline 1 & 0 & -27 & 0.3 & $5.50 \mathrm{E}+02$ & $6.455 \mathrm{E}+02$ & $5.559 \mathrm{E}+02$ & 0 & 0 & 0 \\
\hline 1 & -0.5 & 0.111 & 0.3 & $9.85 \mathrm{E}-01$ & $9.907 \mathrm{E}-01$ & $9.850 \mathrm{E}-01$ & $-4.62 \mathrm{E}-01$ & $-4.624 \mathrm{E}-01$ & $-4.143 \mathrm{E}-01$ \\
\hline 1 & -0.5 & 3 & 0.3 & $5.00 \mathrm{E}-01$ & $5.464 \mathrm{E}-01$ & $3.014 \mathrm{E}-01$ & $-1.79 \mathrm{E}-01$ & $-5.091 \mathrm{E}-02$ & $-1.256 \mathrm{E}-01$ \\
\hline
\end{tabular}


Table A.1 (continued)

\begin{tabular}{|c|c|c|c|c|c|c|c|c|c|}
\hline $\bar{u}_{m}$ & $\Sigma$ & $\vec{p}$ & $n$ & $\overline{\mathrm{Q}}_{\text {sim }}$ & $\bar{Q}_{\text {Bair }}$ & $\overline{\mathrm{Q}}_{\text {new }}$ & $\bar{\tau}_{m ; s i m}$ & $\bar{\tau}_{m ; B \text { aair }}$ & $\bar{\tau}_{m, n e w}$ \\
\hline 1 & -0.5 & 27 & 0.3 & $-5.48 \mathrm{E}+02$ & $-6.435 \mathrm{E}+02$ & $-5.539 \mathrm{E}+02$ & $-1.18 \mathrm{E}-03$ & $-3.062 \mathrm{E}-04$ & $-1.145 \mathrm{E}-03$ \\
\hline-1 & -0.5 & 0.111 & 0.3 & $-1.009 \mathrm{E}+00$ & $-1.009 \mathrm{E}+00$ & $-1.015 \mathrm{E}+00$ & $4.62 \mathrm{E}-01$ & $4.624 \mathrm{E}-01$ & $4.143 \mathrm{E}-01$ \\
\hline-1 & -0.5 & 3 & 0.3 & $-1.50 \mathrm{E}+00$ & $-1.454 \mathrm{E}+00$ & $-1.699 \mathrm{E}+00$ & $1.78 \mathrm{E}-01$ & $5.091 \mathrm{E}-02$ & $1.256 \mathrm{E}-01$ \\
\hline-1 & -0.5 & 27 & 0.3 & $-5.50 \mathrm{E}+02$ & $-6.455 \mathrm{E}+02$ & $-5.559 \mathrm{E}+02$ & $1.18 \mathrm{E}-03$ & $3.062 \mathrm{E}-04$ & $1.145 \mathrm{E}-03$ \\
\hline 0.01 & 0 & 0.333 & 0.5 & $-1.79 \mathrm{E}-02$ & $-1.847 \mathrm{E}-02$ & $-1.798 \mathrm{E}-02$ & 0 & 0 & 0 \\
\hline 0.01 & 0 & 1 & 0.5 & $-7.65 \mathrm{E}-02$ & $-8.276 \mathrm{E}-02$ & $-7.933 \mathrm{E}-02$ & 0 & 0 & 0 \\
\hline 0.01 & 0 & 3 & 0.5 & $-3.33 \mathrm{E}-01$ & $-3.623 \mathrm{E}-01$ & $-3.699 \mathrm{E}-01$ & 0 & 0 & 0 \\
\hline 0.01 & 0 & 9 & 0.5 & $-2.54 \mathrm{E}+00$ & $-2.328 \mathrm{E}+00$ & $-2.640 \mathrm{E}+00$ & 0 & 0 & 0 \\
\hline 0.01 & 0 & 27 & 0.5 & $-2.28 \mathrm{E}+01$ & $-2.285 \mathrm{E}+01$ & $-2.290 \mathrm{E}+01$ & 0 & 0 & 0 \\
\hline 0.01 & 0 & 18 & 0.5 & $-1.01 \mathrm{E}+01$ & $-9.493 \mathrm{E}+00$ & $-1.024 \mathrm{E}+01$ & 0 & 0 & 0 \\
\hline 0.01 & 0.5 & 0.333 & 0.5 & $-1.79 \mathrm{E}-02$ & $-1.847 \mathrm{E}-02$ & $-1.798 \mathrm{E}-02$ & $5.00 \mathrm{E}-03$ & 4.992E-03 & 4.932E-03 \\
\hline 0.01 & 0.5 & 1 & 0.5 & $-7.65 \mathrm{E}-02$ & $-8.276 \mathrm{E}-02$ & $-7.933 \mathrm{E}-02$ & $4.70 \mathrm{E}-03$ & $4.496 \mathrm{E}-03$ & $4.472 \mathrm{E}-03$ \\
\hline 0.01 & 0.5 & 3 & 0.5 & $-3.33 \mathrm{E}-01$ & $-3.623 \mathrm{E}-01$ & $-3.699 \mathrm{E}-01$ & $3.20 \mathrm{E}-03$ & $1.942 \mathrm{E}-03$ & $2.773 \mathrm{E}-03$ \\
\hline 0.01 & 0.5 & 9 & 0.5 & $-2.54 \mathrm{E}+00$ & $-2.328 \mathrm{E}+00$ & $-2.640 \mathrm{E}+00$ & $1.10 \mathrm{E}-03$ & $6.511 \mathrm{E}-04$ & $1.085 \mathrm{E}-03$ \\
\hline 0.01 & 0.5 & 27 & 0.5 & $-2.28 \mathrm{E}+01$ & $-2.285 \mathrm{E}+01$ & $-2.290 \mathrm{E}+01$ & $6.00 \mathrm{E}-04$ & $2.170 \mathrm{E}-04$ & $3.694 \mathrm{E}-04$ \\
\hline 0.01 & 0.5 & 18 & 0.5 & $-1.01 \mathrm{E}+01$ & $-9.493 \mathrm{E}+00$ & $-1.024 \mathrm{E}+01$ & $6.40 \mathrm{E}-04$ & $3.256 \mathrm{E}-04$ & $5.522 \mathrm{E}-04$ \\
\hline 0.1 & 0 & 0.111 & 0.5 & $9.07 \mathrm{E}-02$ & $9.069 \mathrm{E}-02$ & $9.074 \mathrm{E}-02$ & 0 & 0 & 0 \\
\hline 0.1 & 0 & 0.333 & 0.5 & $7.20 \mathrm{E}-02$ & $7.153 \mathrm{E}-02$ & $7.202 \mathrm{E}-02$ & 0 & 0 & 0 \\
\hline 0.1 & 0 & 1 & 0.5 & $1.34 \mathrm{E}-02$ & $7.241 \mathrm{E}-03$ & $1.067 \mathrm{E}-02$ & 0 & 0 & 0 \\
\hline 0.1 & 0 & 3 & 0.5 & $-2.43 \mathrm{E}-01$ & $-2.723 \mathrm{E}-01$ & $-2.799 \mathrm{E}-01$ & 0 & 0 & 0 \\
\hline 0.1 & 0 & 9 & 0.5 & $-2.45 \mathrm{E}+00$ & $-2.238 \mathrm{E}+00$ & $-2.550 \mathrm{E}+00$ & 0 & 0 & 0 \\
\hline 0.1 & 0 & 27 & 0.5 & $-2.27 \mathrm{E}+01$ & $-2.276 \mathrm{E}+01$ & $-2.281 \mathrm{E}+01$ & 0 & 0 & 0 \\
\hline 0.1 & 0 & 18 & 0.5 & $-1.00 \mathrm{E}+01$ & $-9.403 \mathrm{E}+00$ & $-1.015 \mathrm{E}+01$ & 0 & 0 & 0 \\
\hline 0.1 & 0.5 & 0.111 & 0.5 & $9.09 \mathrm{E}-02$ & $9.069 \mathrm{E}-02$ & $9.071 \mathrm{E}-02$ & $4.99 \mathrm{E}-02$ & $4.997 \mathrm{E}-02$ & $4.986 \mathrm{E}-02$ \\
\hline 0.1 & 0.5 & 0.333 & 0.5 & $7.23 \mathrm{E}-02$ & $7.153 \mathrm{E}-02$ & $7.192 \mathrm{E}-02$ & $4.96 \mathrm{E}-02$ & $4.989 \mathrm{E}-02$ & $4.926 \mathrm{E}-02$ \\
\hline 0.1 & 0.5 & 1 & 0.5 & $1.36 \mathrm{E}-02$ & $7.241 \mathrm{E}-03$ & $1.045 \mathrm{E}-02$ & $4.70 \mathrm{E}-02$ & $4.493 \mathrm{E}-02$ & $4.469 \mathrm{E}-02$ \\
\hline 0.1 & 0.5 & 3 & 0.5 & $-2.43 \mathrm{E}-01$ & $-2.723 \mathrm{E}-01$ & $-2.801 \mathrm{E}-01$ & $3.09 \mathrm{E}-02$ & $1.941 \mathrm{E}-02$ & $2.773 \mathrm{E}-02$ \\
\hline 0.1 & 0.5 & 9 & 0.5 & $-2.45 \mathrm{E}+00$ & $-2.238 \mathrm{E}+00$ & $-2.550 \mathrm{E}+00$ & $1.12 \mathrm{E}-02$ & $6.507 \mathrm{E}-03$ & $1.085 \mathrm{E}-02$ \\
\hline 0.1 & 0.5 & 27 & 0.5 & $-2.27 \mathrm{E}+01$ & $-2.276 \mathrm{E}+01$ & $-2.281 \mathrm{E}+01$ & $4.00 \mathrm{E}-03$ & $2.169 E-03$ & $3.694 \mathrm{E}-03$ \\
\hline 0.1 & 0.5 & 18 & 0.5 & $-1.00 \mathrm{E}+01$ & $-9.403 \mathrm{E}+00$ & $-1.015 \mathrm{E}+01$ & $5.80 \mathrm{E}-03$ & $3.254 \mathrm{E}-03$ & $5.522 \mathrm{E}-03$ \\
\hline 0.1 & 1 & 0.111 & 0.5 & $9.12 \mathrm{E}-02$ & $9.069 \mathrm{E}-02$ & $9.061 \mathrm{E}-02$ & $9.97 \mathrm{E}-02$ & $9.975 E 102$ & $9.936 \mathrm{E}-02$ \\
\hline 0.1 & 1 & 0.333 & 0.5 & $7.24 \mathrm{E}-02$ & $7.153 \mathrm{E}-02$ & $7.163 \mathrm{E}-02$ & $9.90 \mathrm{E}-02$ & $9.959 \mathrm{E}-02$ & $9.819 \mathrm{E}-02$ \\
\hline 0.1 & 1 & 1 & 0.5 & $1.35 \mathrm{E}-02$ & $7.241 \mathrm{E}-03$ & $9.785 \mathrm{E}-03$ & $9.39 \mathrm{E}-02$ & $8.969 \mathrm{E}-02$ & $8.918 \mathrm{E}-02$ \\
\hline 0.1 & 1 & 3 & 0.5 & $-2.43 \mathrm{E}-01$ & $-2.723 \mathrm{E}-01$ & $-2.806 \mathrm{E}-01$ & $6.16 \mathrm{E}-02$ & $3.875 \mathrm{E}-02$ & $5.545 \mathrm{E}-02$ \\
\hline 0.1 & 1 & 9 & 0.5 & $-2.45 \mathrm{E}+00$ & $-2.238 \mathrm{E}+00$ & $-2.550 \mathrm{E}+00$ & $2.20 \mathrm{E}-02$ & $1.299 \mathrm{E}-02$ & $2.169 \mathrm{E}-02$ \\
\hline 0.1 & 1 & 27 & 0.5 & $-2.27 \mathrm{E}+01$ & $-2.276 \mathrm{E}+01$ & $-2.281 \mathrm{E}+01$ & $7.37 \mathrm{E}-03$ & $4.330 \mathrm{E}-03$ & $7.387 \mathrm{E}-03$ \\
\hline 0.1 & 1 & 18 & 0.5 & $-1.00 \mathrm{E}+01$ & $-9.403 \mathrm{E}+00$ & $-1.015 \mathrm{E}+01$ & $1.12 \mathrm{E}-02$ & $6.495 \mathrm{E}-03$ & $1.104 \mathrm{E}-02$ \\
\hline 1 & 1 & 0.111 & 0.5 & $9.90 \mathrm{E}-01$ & $9.907 \mathrm{E}-01$ & $9.837 \mathrm{E}-01$ & $8.40 \mathrm{E}-01$ & $8.409 E-01$ & $7.857 \mathrm{E}-01$ \\
\hline 1 & 1 & 0.333 & 0.5 & $9.61 \mathrm{E}-01$ & $9.715 \mathrm{E}-01$ & $9.513 \mathrm{E}-01$ & $8.37 \mathrm{E}-01$ & 8.395E-01 & $7.819 \mathrm{E}-01$ \\
\hline 1 & 1 & 1 & 0.5 & $8.74 \mathrm{E}-01$ & $9.072 \mathrm{E}-01$ & $8.564 \mathrm{E}-01$ & $8.05 \mathrm{E}-01$ & $7.561 \mathrm{E}-01$ & $7.487 \mathrm{E}-01$ \\
\hline 1 & 1 & 3 & 0.5 & $5.95 \mathrm{E}-01$ & $6.277 \mathrm{E}-01$ & $5.615 \mathrm{E}-01$ & $5.88 \mathrm{E}-01$ & $3.266 \mathrm{E}-01$ & $5.365 \mathrm{E}-01$ \\
\hline 1 & 1 & 9 & 0.5 & $-1.58 \mathrm{E}+00$ & $-1.338 \mathrm{E}+00$ & $-1.662 \mathrm{E}+00$ & $2.22 \mathrm{E}-01$ & $1.095 \mathrm{E}-01$ & $2.168 \mathrm{E}-01$ \\
\hline 1 & 1 & 27 & 0.5 & $-2.18 \mathrm{E}+01$ & $-2.186 \mathrm{E}+01$ & $-2.191 \mathrm{E}+01$ & $7.40 \mathrm{E}-02$ & $3.650 \mathrm{E}-02$ & $7.387 \mathrm{E}-02$ \\
\hline 1 & 1 & 18 & 0.5 & $-9.10 \mathrm{E}+00$ & $-8.503 \mathrm{E}+00$ & $-9.251 \mathrm{E}+00$ & $1.11 \mathrm{E}-01$ & $5.475 \mathrm{E}-02$ & $1.104 \mathrm{E}-01$ \\
\hline 0.1 & 0 & 0.111 & 0.75 & $9.07 \mathrm{E}-02$ & $9.050 \mathrm{E}-02$ & $9.075 \mathrm{E}-02$ & 0 & 0 & 0 \\
\hline 0.1 & 0 & 0.333 & 0.75 & $7.21 \mathrm{E}-02$ & $7.071 \mathrm{E}-02$ & $7.217 \mathrm{E}-02$ & 0 & 0 & 0 \\
\hline 0.1 & 0 & 3 & 0.75 & $-1.82 \mathrm{E}-01$ & $-2.100 \mathrm{E}-01$ & $-1.868 \mathrm{E}-01$ & 0 & 0 & 0 \\
\hline 0.1 & 0 & 9 & 0.75 & $-1.03 \mathrm{E}+00$ & $-1.025 \mathrm{E}+00$ & $-1.034 \mathrm{E}+00$ & 0 & 0 & 0 \\
\hline 0.1 & 0 & 27 & 0.75 & $-4.72 \mathrm{E}+00$ & $-4.487 \mathrm{E}+00$ & $-4.732 \mathrm{E}+00$ & 0 & 0 & 0 \\
\hline 0.1 & 1 & 0.111 & 0.75 & $9.12 \mathrm{E}-02$ & $9.050 \mathrm{E}-02$ & $9.070 \mathrm{E}-02$ & $9.97 \mathrm{E}-02$ & $9.988 \mathrm{E}-02$ & $9.978 \mathrm{E}-02$ \\
\hline 0.1 & 1 & 0.333 & 0.75 & $7.26 \mathrm{E}-02$ & $7.071 \mathrm{E}-02$ & $7.204 \mathrm{E}-02$ & $9.95 \mathrm{E}-02$ & $9.982 \mathrm{E}-02$ & $9.939 \mathrm{E}-02$ \\
\hline 0.1 & 1 & 3 & 0.75 & $-1.82 \mathrm{E}-01$ & $-2.100 \mathrm{E}-01$ & $-1.872 \mathrm{E}-01$ & $8.36 \mathrm{E}-02$ & $7.287 \mathrm{E}-02$ & $8.215 E-02$ \\
\hline 0.1 & 1 & 9 & 0.75 & $-1.02 \mathrm{E}+00$ & $-1.025 \mathrm{E}+00$ & $-1.034 \mathrm{E}+00$ & $6.55 \mathrm{E}-02$ & $5.062 \mathrm{E}-02$ & $6.009 \mathrm{E}-02$ \\
\hline 0.1 & 1 & 27 & 0.75 & $-4.72 \mathrm{E}+00$ & $-4.487 \mathrm{E}+00$ & $-4.732 \mathrm{E}+00$ & $4.28 \mathrm{E}-02$ & $3.510 \mathrm{E}-02$ & $4.196 \mathrm{E}-02$ \\
\hline
\end{tabular}




\section{References}

[1] Bair SA. Reynolds-Ellis equation for line contact with shear-thinning Tribology International 2002;39:310-6.

[2] Chapkov AD, Bair S, Cann P, Lubrecht AA. Film thickness in point contacts under generalized Newtonian EHL conditions: numerical and experimenta analysis. Tribology International 2007;40:1474-8.

[3] Bair S, Khonsari MM. Generalized Reynolds equations for line contact with double-Newtonian shear-thinning. Tribology Letters 2005;18:513-20.

[4] Tao J, Hughes TG, Evans HP, Snidle RW, Hopkinson NA, Talks M, et al. Elastohydrodynamic lubrication analysis of gear tooth surfaces from micropitting tests. Journal of Tribology 2003;125:267-74.

[5] Dowson D, Taylor CM, Zhu G. A transient elastohydrodynamic lubrication analysis of a cam and follower. Journal of Physics D: Applied Physics 1992;25:A313-20.

[6] Greenwood JA. Two-dimensional flow of a non-Newtonian lubricant. Proceedings of the Institution of Mechanical Engineers Part J 2000;214:29-41.

[7] Kim HJ, Ehret P, Dowson D, Taylor CM. Thermal elastohydrodynamic analysis of circular contacts part 2: non-Newtonian model. Proceedings of the Institution of Mechanical Engineers Part J 2001;215:353-62.

[8] Tao J, Hughes TG, Evans HP, Snidle RW, Hopkinson NA, Talks M, et al. Elastohydrodynamic lubrication analysis of gear tooth surfaces from micropitting tests. Journal of Tribology 2003:125:267-74

[9] Holmes MIA., Qiao H, Evans HP, Snidle RW, Surface contact and damage in micro-EHL. Life cycle tribology. In: Tribology and interface engineering series vol. 48; p. 605-16-Proceedings of the 31st Leeds-Lyon symposium on tribology 2005held at Trinity and All Saints College, Horsforth, Leeds, UK. 7th-10th September 2004.

[10] Sharif KJ, Kong S, Evans HP, Snidle RW. Contact and elastohydrodynamic analysis of worm gears part 1: theoretical formulation. Proceedings of the Institution of Mechanical Engineers Part C 2001;215:817-30.
[11] Ehret P, Dowson D, Taylor CM. On lubricant transport conditions in elastohydrodynamic conjunctions. Proceedings: Mathematical, Physical and Engineering Sciences 1998;454(1971):763-87.

[12] Kumar P, Khonsari MM. On the role of lubricant rheology and piezo-viscous properties in line and point contact EHL. Tribology International 2009;42: $1522-30$.

[13] Bair S. High pressure rheology for quantitative elastohydrodynamics. Tribology and interface engineering series, 54. Elsevier; 2007.

[14] Carreau PJ. Rheological equations from molecular network theories 1972;16(1):99-127Transactions of the Society of Rheology 1972;16(1): 99-127.

[15] Bair S, Vergne P, Querry MA. Unified shear-thinning treatment of both film thickness and traction in EHD. Tribology Letters 2005;18(2):145-52.

[16] Bair S, Khonsari MM. Reynolds equation for common generalized Newtonian model and an approximate Reynolds-Carreau equation. Proceedings of the Institution of Mechanical Engineers Part J 2006;220(4):365-74.

[17] Eric W "Hypergeometric function". In MathWorld-a Wolfram web resource. 〈http://mathworld.wolfram.com/HypergeometricFunction.html〉.

[18] Abramowitz M, Stegun IA. Handbook of mathematical functions. New York: Dover Publications; 1970 Ed.

[19] Lin JR. Non-newtonian effects on the dynamic characteristics of onedimensional slider bearings: Rabinowitsch fluid model. Tribology Letters 2001;10(4):237-43.

[20] Liu Y, Wang Q, Bair S, Vergne P. A quantitative solution for the full shearthinning EHL point contact problem including traction. Surface contact and damage in micro-EHL. Tribology Letters 2007;28:171-81.

[21] Holmes MJA, Qiao H, Evans HP, Snidle RW. Surface contact and damage in micro-EHL. Tribology and interface engineering series 2005;48:605-16. 